\title{
MedienPädagogik
}

Zeitschrift für Theorie und Praxis der Medienbildung

\section{Stellungnahme des Vorstands der Sektion Medienpädagogik der DGfE zur Covid-19 Situation}

\author{
Digitale Medien in Bildung und Erziehung: Krisenzeiten verdeutlichen \\ Defizite und Innovationspotenziale
}

Klaus Rummler, Sandra Aßmann, Patrick Bettinger und Karsten D. Wolf

Im Zuge der Corona-Krise wurde das Lehren und Lernen mit digitalen Medien in unterschiedlichen Bildungsbereichen unvermittelt zum primären - und oft auch einzigen - Mittel der Wahl. Quer durch alle Altersgruppen und gesellschaftlichen Felder sah man sich vor der Herausforderung, unterschiedliche Formate sozialer Interaktion in kürzester Zeit durch den Einsatz digitaler Medien kompensieren zu müssen. Nach mittlerweile vier Monaten «Lockdown» und Konsequenzen zeigen die Debatten um Lehren und Lernen mit digitalen Medien deutlich die Heterogenität der Positionen. Das Spektrum reicht von kompletter Ablehnung bis hin zu Forderungen, die gegenwärtigen digitalen Lehr-Lern-Praxen in unterschiedlichen bildungsinstitutionellen Kontexten als ideale Blaupause heranzuziehen. Wir plädieren für eine differenzierte Analyse, warnen aber insbesondere davor, mit einer hoffentlich zunehmenden Normalisierung des öffentlichen Lebens einfach wieder zum alten Status Quo zurückzukehren: Trotz der teils dramatischen - und weitgehend noch nicht absehbaren - Auswirkungen der Corona-Krise sind Innovationsimpulse in unterschiedlichen Bildungsbereichen zu erkennen. Insbesondere das System Schule wurde in diesem Zusammenhang in Bewegung gebracht. Ohne jahrzehntelang entwickelte Erkenntnisse und Konzepte durch ad-hoc Improvisationen ersetzen zu wollen und die negativen Auswirkungen zu schmälern, sehen wir in den Entwicklungen der letzten Monate auch Chancen, die Digitalisierung im Bereich der Bildung und Erziehung produktiv gestalten zu können.

Deshalb wollen wir auf Basis medienpädagogischer Forschung und Entwicklung folgende drei zukunftsgerichtete Empfehlungen und Forderungen formulieren:

- In medienpädagogische Aus- und Fortbildung investieren: Die in einer Krisenzeit improvisierten Handlungen ersetzen keine geplanten und wissenschaftlich begründeten Strategien. Der Digitalisierungsimpuls ist über die bereitgestellten Mittel hinaus nachhaltig zu finanzieren. Dies beinhaltet neben Hardware, Software, Content und Support vor allem Aus- und Fortbildung von Lehrpersonen, Pädagoginnen und Pädagogen sowie eine systematische und professionell unterstützte Konzeptentwicklung. Medienpädagogische Studiengänge bzw. medienpädagogische Anteile in pädagogischen Studiengängen sind gerade vor dem Hintergrund der jüngsten Erfahrungen weiterzuentwickeln (vgl. https://doi.org/10.21240/mpaed/00/2017.12.04.X). Dazu bedarf es u. a. in den erziehungswissenschaftlichen und fachdidaktischen Studienanteilen der fachwissenschaftlichen Auseinandersetzung mit digitalen Medien. Nur so ist eine professionelle Gestaltung einer digitalen Transformation der Bildung zu leisten. 
- Das Potenzial der Vernetzung von Bildungseinrichtungen und ausserschulischer Medienpädagogik stärken: Die gegenwärtigen und zukünftigen Herausforderungen sind nicht alleine von staatlichen Bildungsinstitutionen wie der Schule zu bewältigen. Sehr gut qualifizierte Medienpädagoginnen und Medienpädagogen in ausserschulischen Institutionen bieten ein reichhaltiges Angebot lebensnaher und generationenübergreifender Bildungsangebote. Diese sind über die bisherigen Kooperationen stärker einzubinden, finanziell zu stärken und inhaltlich weiterzuentwickeln, um nachhaltige Vernetzungsstrukturen zu schaffen.

- Medienbildung für ein ganzes Leben gestalten: Die Corona-Pandemie hat noch einmal deutlich gemacht, dass nicht nur Schülerinnen und Schüler sowie Lehrpersonen, sondern auch Eltern oder Grosseltern bzw. Arbeitnehmerinnen und Arbeitnehmer über ein hohes Mass an Medienkompetenz verfügen müssen, um mit veränderten Rahmenbedingungen umzugehen und sich Medien sinnvoll für eine aktive Teilhabe an der Gesellschaft anzueignen. Medienbildungsangebote sind somit über die gesamte Lebensspanne anzubieten und auszubauen, um soziale Ungleichheiten abzubauen und die medienpädagogischen Potenziale zur Stärkung von Diversität in allen Lebensbereichen fruchtbar zu machen sowie dazu konsequent Spielräume der Gesetzgebung und in der Verwaltung zu nutzen.

Medienbildung trägt dazu, neben einer rein anwendungsbezogenen Perspektive (Wie nutze ich das?) und einer technologischen Perspektive (Wie funktioniert das?), eine gesellschaftlich-kulturelle (Wie wirkt das?) sowie eine emanzipatorisch-bildungstheoretische Perspektive (Was will ich?) bei.

Schliesslich möchten wir als Vorstand der Sektion Medienpädagogik darauf aufmerksam machen, dass sich gerade wissenschaftliche Qualifikandinnen und Qualifikanden in Anbetracht der gegenwärtigen Krise in einer Lage befinden, die noch weit mehr als bisher schon als prekär bezeichnet werden muss. Insbesondere empirische Forschungsprojekte sind zum Teil schon seit mehreren Monaten nicht mehr durchführbar, oft kulminieren verschiedene Verzögerungen zu Konstellationen, die eine Weiterführung oder einen Abschluss von Qualifikationsarbeiten gefährden. Wir möchten daher zum einen alle Kolleginnen und Kollegen, die Betreuungsaufgaben wahrnehmen, dazu auffordern, den je individuellen Situationen der Qualifikandinnen und Qualifikanden bestmöglich Rechnung zu tragen und Entlastung zu schaffen. Zum anderen appellieren wir aber auch an Universitäten und Drittmittelgebende, zusätzliche Mittel zur Verlängerung von Beschäftigungsverhältnissen oder Stipendien bereitzustellen und Spielräume in Gesetzgebung und Verwaltung konsequent zu nutzen.

Die gegenwärtige gesellschaftliche Lage könnte nicht deutlicher vor Augen führen, wie wichtig eine fundierte und differenzierte Auseinandersetzung mit Fragen von Lehren, Lernen, Bildung, Erziehung und Sozialisation im Kontext der digitalen Transformation ist. Die Heterogenität der Situationen und Praxen sollte dabei berücksichtigt werden. Nur wenn medienpädagogische Perspektiven im Bildungsbereich umfassend Berücksichtigung finden und ein unvoreingenommener Diskurs um Chancen und Risiken digitaler Medien zustande kommt, kann Medienbildung gelingen. 